\title{
New developments in pervasive and ambient information systems
}

\author{
Elhadi Shakshuki • Muhammad Younas
}

Published online: 28 April 2012

(C) Springer-Verlag London Limited 2012

This special issue contains selected papers from the 2nd International Conference on Ambient Systems, Networks and Technologies (ANT 2011), which was held at Niagara Falls, Ontario, Canada, September 19-21, 2011. The conference attracted a large number of scientific papers that contributed to the state-of-the-art in the areas to the theme of the conference. All the papers selected for this special issue were subjected to a two rounds of rigorous review process. Based on the reviewers' feedback, a total of seven papers were selected for publication from ten invited papers. The accepted papers cover interesting works on new developments in pervasive and ambient systems such as smart environments, environmental monitoring, information search and processing, and the practical use of smart devices in industrial applications. A brief summary is as follows.

Identifying and recognizing objects in (indoor) smart environment is one of the challenging research areas in pervasive and ambient systems. The paper by Menon et al. proposes a novel model for identifying and tracking objects in smart environments. This work integrates recognition and spatio-temporal reasoning in order to achieve improved precision in smart environments. The proposed approach is evaluated through experimentation in terms of face recognition, which shows improved performance, precision, and recall.

E. Shakshuki $(\bowtie)$

Jodrey School of Computer Science, Acadia University,

Wolfville, NS, Canada

e-mail: elhadi.shakshuki@acadiau.ca

\section{Younas}

Department of Computing and Communication Technologies, Oxford Brookes University, Oxford, UK

e-mail: m.younas@brookes.ac.uk
Wireless sensor network (WSN) augmented with ambient intelligence has interesting applications in home living. Rawi et al. in their paper develop WSN-based Human Comfort Ambient Intelligence system, which exploits fuzzy rules in order to measure human comfort index in a living space. It explores complex relationship among multiple comfort factors such as thermal, visual, and indoor air comfort factors. Further, the system is developed in a way such that it provides flexibility in terms of adding or removing comfort factors as well as sensor nodes.

Trust is another important issue in providing truly smart environments in living spaces. El Husseini et al. propose a trust model that takes into account user's past and present behavior. The proposed model utilizes a lightweight authentication key agreement protocol in order to ensure trust and to provide mutual authentication between communicating entities. The model is developed such that it optimizes the communication and processing of resource scare devices in smart environments.

Al-Turjman et al. explore the utilization of WSN in environmental monitoring applications such as forestry. The large number of failures complicates the process of environmental monitoring as WSNs often suffer from physical damages and network partition. This paper proposes 3D grid-based deployment for relay nodes and is based on a minimum spanning tree construction. It is used in re-connecting the disjointed WSN sectors and has the potential to optimize various factors including: average relay node count and distribution, the scalability of the federated WSNs in large-scale applications, and the robustness of the topologies.

Effective processing of queries in mobile ad hoc network is crucial in order to optimize performance, reduce energy consumption, and avoid failures. Nghiem et al. 
propose a querying processing system in mobile $\mathrm{P} 2 \mathrm{P}$ environment. The proposed approach aims at the search and validation algorithm for $\mathrm{kNN}$ queries. The objective is to reduce energy consumption by sharing of data through peers instead of centralized data sharing. The proposed approach is shown to save communication cost as well as optimize the network latency involved in query processing.

Iwata et al. tackle the issue of content search in mobile setup by taking into account the current context and user's activity. The authors design and develop a system that helps users in content search. The system is developed in a way that runs in two different modes: location-based and menu-based content search. The former is activated when the user is stationary, while the latter is activated when the user is moving. The system is evaluated through usability experiments, which shows that users can search required information more easily as compared to the classical search systems.
Providing high level of security on current WSNs platforms is unattainable, especially against attacks based on node compromise. Moh'd et al. propose SN-SEC, a 32-bit RISC secure wireless sensor platform with hardware cryptographic primitives. The aim of this approach is to secure holes in current WSNs platforms and compare the main approaches to implementing their cryptographic primitives in terms of security, time, and energy efficiency.

The guest editors would like to take this opportunity to thank the authors for the efforts they put in the preparation of their manuscripts and for their valuable contributions. We wish to express our deepest gratitude to the referees for their thorough and detailed reviews. Last, but not least, our sincere thanks go to the Editor-in-Chief, Dr. Peter Thomas, of the Journal of Pervasive and Ubiquitous Computing for the exceptional support and assistance he provided throughout this process. 